\title{
On the Pathogenesis of Autoimmune Mechanism in Gastritis
}

\author{
Takashi KATAYAMA \\ The First Department of Internal Medicine, \\ Tokyo Medical and Dental University
}

After the oral administration of $0.1 \mathrm{~N} \mathrm{NaOH}$ to DD-strain mice, a gastric antigen was detected within 24 days and antigastric antibody in about 10 days. The similar findings might be expected in man, so the following immunological studies were: performed in 185 cases of human chronic gastritis. Antihuman gastric rabbits serum was used for detecting gastric antigen and immunofluorescence for parietal cell antibody (PCA). Gastric antigen was positive in 50 cases and PCA in 54 . Both antigen and antibody were positive in 16 , and all of them revealed marked infiltration of immunoglobulin-containing cells into the mucosal layer. Above all more than half of these 16 showed hypo- or anacidity and a decrease in excretion of intrinsic factor. Cases more than 50-year-old were found to have a decrease in number of parietal cells as well as in gastric excretion. Three of 16 showed a decrease in intrinsic factor as pernicious anemia. Complement-binding antibody or antithyroid antibody was recognized in several cases, but these antibodies have no relation between GA as well as PCA. There were no histological and functional differences between positive PCA cases and negative. These results suggested that gastric autoimmunity is produced in association with damages on gastric mucosa, and there is a certain type of gastritis, accompanying autoimmune phenomenon as well as progressing gastric atrophy. But PCA might not be acting as the mucosal damaging agent. Therefore, it is speculated that the genetic factor would be related rather to susceptibility of gastric mucosa to the autoimmune mechanism, than to the process which produces the autoimmunity. 